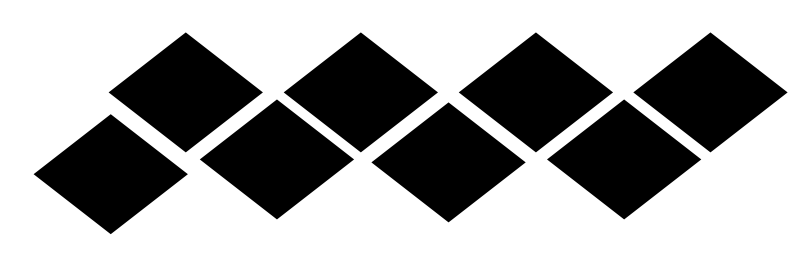

\title{
Hétérodoxie dans la poésie engagée moderne: Eluard et Matar comme modèles (étude analytique)
}

\begin{abstract}
Dr.
Amal Abdel-Sattar Abd-Allah Abdel-Karim

Maître de conférences au département de français

Faculté des langues Al-Alsun, Université de Minia
\end{abstract}

DOI: 10.21608/qarts.2021.95478.1228

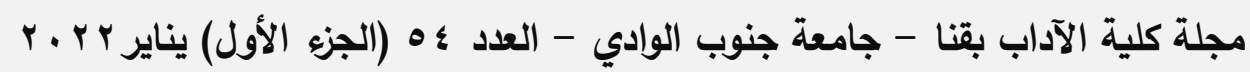

ISSN (Print): 1110-614X الترقيم الدولي الموحد للنسخة المطبوعة

ISSN (Online): 1110-709X الترقيم الدولي الموحد للنسخة الإكترونية الموحية

https://qarts.journals.ekb.eg

موقع المجلة الإكتروني: 


\title{
Hétérodoxie dans la poésie engagée moderne:
}

\section{Eluard et Matar comme modèles (étude analytique)}

\author{
Prepared by \\ Dr. Amal Abdulsattar Abdullah Abdelkarim \\ Maître de conférences au département de français \\ Faculté des langues Al-Alsun, Université de Minia \\ uni.aurore2@gmail.com
}

\section{Résumé}

La littérature, et surtout la poésie engagée, avait sa manière de défendre des causes et adopter des principes soient religieux, soient patriotiques, philosophiques ou idéologiques en général. Le langage utilisé est d'une valeur qui varie selon le discours dirigé. Le surréalisme, comme tendance littéraire et poétique ne s'attarde pas à mettre son grain de sel dans le courant engagé. Il a utilisé ses propres moyens, son propre langage pour renvoyer son message sublime au lecteur, sans considération de l'occultisme de ce message et de l'inaccessibilité de ses données. Dans cette recherche, nous prétendons avoir accès à certains points obscurs de ce monde par l'aide des clés dont utilisaient les poètes surréalistes; et nous allons découvrir les moyens dont disposaient ces poètes pour renvoyer leur parole et s'adhérer au camp des résistants en faveur de leurs patries. La forme et le fond des poèmes contribuent fortement à nous transposer ce monde ambigu et clos. La métrique, la versification et les figures de style; tout cela n'était pas gratuit, même s'il s'avère le contraire. Le poète surréaliste, par une poésie rigoureuse et incompréhensible dans ses détails, pouvait s'aligner en tant qu'homme de principes et résistant par excellence.

Mots clés: Poésie engagée, surréalisme, Eluard, Afifi Matar, poésie hermétique, occultisme poétique. 


\section{Introduction}

Dans la littérature mondiale, il y a des tendances qui témoignent de l'intérêt au souci humain et à la souffrance commune. La littérature engagée en découle pour démontrer que les hommes de lettres partagent aussi les mêmes malheurs et soucis. Et comme la poésie moderne est l'un des genres littéraires, les poètes ne s'attardent pas à y mettre leur empreinte. La poésie a déjà ses modes d'expression dont les écoles poétiques donnent la preuve. Si l'école surréaliste aux années vingt et après cela s'avérait comme une voix violente et insolite, elle mobilisait aussi des poètes engagés pour défendre leur parole contre la tyrannie et l'injustice en faveur de la justice et de l'égalité des hommes. Paul Eluard, par ses deux poèmes Liberté et La victoire de Guernica avait donné l'exemple de cet engagement dans le camp de sa nation contre l'occupation nazie. D'un autre côté, le poète égyptien révolutionnaire Mohamed Afifi Matar avait écrit plusieurs recueils durant sa carrière poétique, dont nous avons choisi L'ancienne terre "al:ard alqadima" et Cette nuit "haadhihi allaïla", qui répondent, tous les deux, à un engagement de la part du poète envers sa patrie contre l'injustice et la tyrannie. Les deux voix, orientale et occidentale, se rencontrent dans certains points communs dont l'engagement politique et social, la souffrance, l'emploi d'un langage ésotérique pour s'exprimer, ...etc. Autour de ce dernier point, notre recherche s'axe. Chez les deux poètes nous trouvons ce langage poétique hermétique qui provient soit d'un impact surréaliste chez Eluard, soit d'un ésotérisme verbal chez Matar. Les deux poètes nous semblent d'emblée incompréhensibles, ce qui donne à leur poésie une éternité inexplicable. 
Paul Eluard (1895-1952) est un poète français renommé, l'un des fondateurs du mouvement surréaliste et il était le premier à ouvrir la voie à une action artistique politiquement engagée auprès du parti communiste. Parmi ses œuvres les plus notoires Capitale de la douleur, La victoire de Guernica, Poésies et vérité et Liberté.

Mohamed Afifi Matar (1935-2010), un poète égyptien militant; il a quitté l'Egypte pour l'Irak à cause des difficultés avec le régime militaire sous le règne de Sadate. Il y est vécu plusieurs années avant de se retourner en Egypte. "L'établissement culturel égyptien l'a délibérément marginalisé en raison de ses positions politiques en opposition au pouvoir. Il a publié dans la revue Sanabel (1969-1972), qu'il éditait avec un groupe de ses amis par leurs propres efforts, un poème intitulé 68 dans lequel il invitait les étudiants à manifester pour mettre fin à la période de ni paix ni guerre qui a marqué les débuts du règne de Sadate". (ZAKAREYA 2014).

Comme son prédécesseur Sadate, le règne de Moubarak ne lui était pas mieux. Le fait de son adhésion au parti Baas égyptien était la cause de son emprisonnement accusé d'implication, avec d'autres personnes, dans un complot antigouvernemental en avril 1991. Matar est reconnu comme l'un des poètes arabes modernes les plus difficiles: "Afifi Matar est considéré comme l'un des poètes les plus importants de la génération littéraire des années soixante au niveau du monde arabe, et à la tête des poètes innovateurs dans le poème arabe" (AZZARRA 2020). Le poème de Matar "n'est pas similaire à d'autres poèmes, il n'est ni copié ni imité, mais il est plutôt rebelle et rebelle à tout le reste, comme s'il s'agissait d'une marque" (AZZARRA 2020). 
Soureyya Lemjadi indique la différence entre la poésie ancienne et la poésie nouvelle en ce qui concerne le langage poétique. Le poème ancien était principalement basé sur le style pompeux, l'emploi de la métaphore et de l'analogie, tandis que le poème moderne se débarrasse du poids de la rhétorique, et dépend de la structuration systématique et urgente du langage. (LEMJADI 2011)

Parmi ses recueils notons La faim et la lune (aljou'e oualqamar), Le limon parle (yatahaddathu attami), Le quatrain de la joie (roubayyeat al-farah), La cérémonie de la momie sauvage (ihtifaaliyat al-momia al-motaouahhecha), ... etc.

Son écriture se caractérise de l'inspiration de la philosophie grecque, de l'héritage arabo-islamique et du patrimoine folklorique. (EL-KAFRAOUI 2020)

\section{La problématique}

Partant du fait que la poésie engagée porte un message, nous nous inclinons à montrer que ce message ne devrait pas être toujours à la portée des lecteurs. Les poètes engagés, dont Eluard et Afifi Matar, tendaient à envelopper leur poésie d'une sorte d'occultisme très cher aux surréalistes. Le message se brouille et le lecteur se trouve obligé de déployer un effort pour décortiquer le texte poétique afin de le comprendre.

Le langage poétique surréaliste se dévie du chemin parcouru par les écrivains, et surtout les poètes engagés, pour inventer un langage propre, plus ésotérique, moins accessible et rarement saisi. 
Cette recherche prétend répondre aux questions suivantes: pourrait-on classer la poésie surréaliste en tant qu'engagée? Autrement dit, comment et par quels moyens le langage poétique occulte ou énigmatique pourrait servir de moyen de lutte contre les maux de la société? Et, jusqu'à quel point les poètes surréalistes engagés pouvaient changer les critères de la poésie engagée?

\section{La méthode suivie}

Du fait que notre travail sera consacré à l'étude des modèles de la poésie surréaliste occidentale et orientale, donc la méthode la plus convenable sera la méthode analytique. Nous allons démarrer de l'étude de la forme et puis l'étude du fond; les mots, les phrases, les figures de style, ...etc.

\section{Justification du choix}

Notre choix de ces poètes, Eluard et Afifi Matar, procède d'une estime pour la poésie engagée. Cet intérêt se redouble d'un besoin de conquérir le monde des poésies obscures dont abondent l'école surréaliste française et arabe et que représentent ces deux poètes qui réunissent un patriotisme distingué et un langage hermétique indocile à la compréhension immédiate.

Le choix des poèmes donne l'exemple de la poésie engagée. Ces poèmes sont proches dans l'idée, célèbres et emblématiques. Leur engagement est politique: pour Eluard, nous avons choisi Liberté et La victoire de Guernica. Pour Afifi Matar nous avons choisi L'ancienne terre et Cette nuit. Des points communs sont partagés entre ces poèmes: soit dans le but pour lequel ils sont écrits, soit dans la philosophie prédominante, soit dans le langage utilisé. 


\section{Définitions:}

\section{La poésie engagée}

La tendance poétique ne cessait pas depuis longtemps de se bifurquer en deux voies distinctes: la poésie lyrique et la poésie engagée. Dès le moment où l'on reproche aux poètes de rester dans leurs tours d'ivoire et de s'enfoncer dans leur rêverie, un courant naît pour prouver que l'art pourrait se mêler aux problèmes de la société et aspirerait à en trouver des solutions ou au moins à en partager le souci.

Les représentants du premier courant de poètes sont nombreux ; on en signale leur chef de file Théophile Gautier ou les parnassiens qui voient qu'un principe de "l'art pour l'art" suffit à être un but en lui-même. Leur argument à ce propos est que la poésie est faite pour être belle; puisque la beauté est un objectif en elle-même. Des poètes éminents comme Baudelaire leur partagent ce point de vue.

Les représentants du deuxième courant, les poètes engagés, au contraire, voient que la beauté de la poésie ne doit pas rompre avec les problèmes de la société et des gens qui lisent cette poésie. Le poète devrait prendre parti dans une cause sociale, morale, philosophique, religieuse ou politique. C'est pour cela l'art s'éternise et se présente comme authentique. Hugo, Aragon et Eluard sont des phares dans cette tendance.

Si l'on trace historiquement les empreintes des poètes engagés, on trouve des poètes comme d'Aubigné et Ronsard dans leur engagement religieux au seizième siècle. André Chénier dans son engagement politique au dix-huitième siècle, Victor Hugo dans son engagement politique au dix-neuvième siècle; et ce 
courant arrive à son apogée avec l'apparition du surréalisme avec des poètes comme René Char, Louis Aragon, Robert Desnos et Paul Eluard.

\section{La poésie surréaliste}

Selon le dictionnaire le Petit Robert, le surréalisme est un "ensemble de procédés de création et d'expression utilisant toutes les forces psychiques (automatisme, rêve, inconscient) libérées du contrôle de la raison et en lutte contre les valeurs reçues; mouvement intellectuel révolutionnaire affirmant la supériorité de ces procédés." (ROBERT 1993). Il est né en 1924 avec la publication du premier Manifeste du surréalisme d'André Breton.

Marie-Paule Berranger voit que "du romantisme et du symbolisme, le surréalisme hérite le pressentiment fasciné de l'invisible. Devant le rideau et derrière le miroir, il cherche le point ou pourraient coexister le grand jour et la grande nuit". (BERRANGER 1997, 77)

Les différentes inspirations dont disposaient les écrivains surréalistes pour réaliser l'objectif de leur tendance sont maintes. On en compte "l'écriture automatique, les sommeils hypnotiques, les récits de rêve, l'écoute des phrases de demi sommeil, les simulations des discours de la folie, la mise en circulation d'objets oniriques et d'objets à fonctionnement symbolique, la quête de la trouvaille, ..." (BERRANGER 1997, 96).

La courant surréaliste en poésie arabe égyptienne se complique du point de vue générique: Afifi Matar, considéré comme chef de file de cette tendance poétique, devrait se créer des outils propres qui le distingue des autres poètes de son époque et 
qui l'éloigne d'être copiste d'une tendance occidentale très à la mode à l'époque.

L'occultisme en poésie n'a pas de public, ni d'amateurs parmi les intellectuels qui sont habitués à la poésie claire: "On retrouve que Matar fait exploser les énergies de la langue avec un vocabulaire qui peut paraître étrange au texte poétique, qui tout au long de son histoire - à l'exception de la poésie préislamique - s'est caractérisé par une simplicité et une facilité dans l'acquisition du vocabulaire. Un vocabulaire qui confirme le pouvoir de la langue arabe dans la formation, la dérivation et la génération". (HAMDI 2018). Du point de vue thématique, 'homme était le souci du poète: "dans l'œuvre de Mohamed Afifi Matar, vous êtes face à un poète qui n'a pas à imposer les détails de sa vie et de son parcours social et politique au texte, mais il se présente plutôt dans le texte comme un être humain satisfait de l'acte humain que des millions de personnes lui partagent [donc] la caractéristique la plus importante de la poésie de Muhammad Afifi Matar est l'humanisme." (HAMDI 2018).

\section{Choix du corpus}

Le fait de choisir les titres d'une œuvre poétique subit une exigence suggestive de la part du poète résumant son exercice langagier dans l'écriture poétique. Les poètes surréalistes le font avec beaucoup de soin: d'une part, le titre introduit à l'aventure langagière que le lecteur va mener, d'une autre part ce même titre avertit le lecteur contre le piège de la perte dans l'occultisme du texte poétique. Pour Eluard, ce jeu lui tient au cœur: Liberté et La victoire de Guernica sont des titres illustres qui ont éternisé ces deux poèmes. Liberté est un seul mot dont la connotation dépasse dans le texte son sens ordinaire. L'absence de la ponctuation dans 
le poème traduit pratiquement cette liberté. Le manque aussi de la rime et du mètre musical et rythmique est un écho du sens de ce mot. Le titre introduit le lecteur dans un monde pareil au seuil d'où on y entre. Pour le deuxième poème, La Guernica, il a été inspiré par un fait historique. Le 26 avril 1937, les avions de l'armée allemande ont bombardé Guernica, la ville espagnole, pendant trois heures; faisant un bilan de deux mille morts. Les atrocités de la guerre sont dénoncées d'abord de la part d'un être humain, et ensuite d'un poète militant. Le titre ne donne qu'un jeu de rhétorique qu'on appelle l'antiphrase. Cette dernière exprime une "manière d'employer un mot, une locution dans un sens contraire au sens véritable, par ironie ou euphémisme." (ROBERT 1993). C'est donc exprimer une idée par son contraire dans une intention ironique: Guernica était vaincu et non pas vainqueur. L'image de la victoire, même renversée dans le poème, présente la vraie victoire de l'existence humaine contre les aspects de la mort et la machine guerrière. Le poète ne se moque jamais de cet évènement, il se moque plutôt du meurtrier en dénonçant sa criminalité.

Mohamed Afifi Matar n'est pas moins significatif à ce propos: Cette nuit, (hadhihi allailla), est une locution nominale composée de l'adjectif démonstratif féminin du singulier et le substantif, un nom féminin singulier. Les connotations du mot nuit entraine cette jonchée de significations négatives concernant la défaite, l'injustice, la soumission et l'humiliation.

Son deuxième poème intitulé L'ancienne terre, (al'ard alqadima), nous renvoie à une atmosphère légendaire. Ce poème tisse ce lien très fort entre la terre et l'héritage, l'identité humaine sur la terre des ancêtres et cette relation directe entre eux; donc plus l'homme oublie son héritage, plus il se déracine de sa terre. 
Ce poème a été écrit comme une réponse à la normalisation avec Israël, avec qui l'Egypte avait entretenu une guerre durant six ans. L'apparition du poème avait renvoyé son auteur à la geôle et puis à l'exil.

\section{Structure des poèmes}

La structure des poèmes reflète aussi l'état de désordre renvoyé par les thèmes et l'état d'âme des auteurs. La victoire de Guernica se compose de quatorze strophes numérotées par des chiffres romains, que chacun pourrait contenir un vers, deux vers ou quatre vers épars sans ordre ni gradation. Le thème principal, qui est les horreurs de la guerre, ne laisse le lecteur sans le faire réfléchir à la destruction causée par la guerre et le chaos produit même dans la forme poétique du texte.

Liberté se compose de vingt-et-une strophes, en forme de quatrains, trois heptasyllabes suivis d'un refrain en tétrasyllabe, "j'écris ton nom", qui ne change qu'à la strophe finale.

Les poèmes de Matar sont en vers libres, sans une forme précise. L'ancienne terre contient environ cinquante-neuf vers qui varient en longueur entre un seul mot et une phrase. Cette nuit contient vingt-cinq vers de longueur variée, dont le plus court contient deux mots, et le plus long contient une phrase complète.

\section{Métrique des poèmes}

Le mètre dans chaque poème du corpus n'est pas équilibré. Le fait de choisir deux modèles de poètes surréalistes nous mène à comprendre la préférence du vers libre pour chacun d'eux. Le refus du vers traditionnel chez les deux poètes répond à un besoin de libération du langage poétique des contraintes prosodiques, ainsi que des contraintes politiques et sociales. 
Les vers libres sont des vers qui ne riment pas forcément, inégaux dans le nombre de syllabes et n'obéissent pas à une rythmique fixe. Ces vers produisent leur propre musique des rimes intérieures, des assonances ou des allitérations. ${ }^{1}$

En revanche, nous trouvons que l'appellation en poésie arabe de ce genre commençait par l'expression de chi'r morsal, cela égalerait le "vers blanc".

A ce propos, Yusuf Ad-Dike constate que les débuts de ce genre de poésie "[avait] commencé dès le début du siècle dernier sous le nom de chi'r morsal, ou de poésie libre. Dans les années quarante cela s'appelait la nouvelle poésie ou ch'ir at-taf'ila. Après les années cinquante on l'a appelé le vers libre. Parmi les nominations étranges suggérées par les critiques, celle de Dr, Ihsan Abbas qui l'a nommé ghosn, ou le rameau; un nom inspiré par la nature et non de l'art, car cette poésie contient en elle-même une variation naturelle de longueur comme c'est le cas avec les branches de l'arbre, et que l'arbre a un rôle important dans les symboles, les rituels, les attitudes humaines et les similitudes artistiques". (AD-DIKE 2021). A cette gamme de dénominations, s'ajoute celle de chi'r manthour, ou poème en prose, une forme poétique qui avait rompu avec la poésie traditionnelle. ${ }^{2}$

Avec Le groupe Apollo, fondé en 1932, on n'a pas eu de principes précis concernant le courant littéraire auquel on s'attache; mais cela représentait la rupture partielle avec l'école poétique traditionnelle représentée dans les poètes de madrasat alba'th, l'école da la Résurrection. Ahmad Zaki abou Chādi était le père du courant Apollo qui a choisi Ahmad Chawqi comme chef de ce mouvement. Après la mort de Chawqi, Khalil Mûtrān y a été élu, et puis Abou Chādi. Les membres de ce groupe ont publié 
leur magazine intitulé Apollo qui est considéré un véritable manifeste des concepts et des productions de ce courant poétique. Le mouvement Apollo a profondément influencé la poésie arabe moderne, bien qu'il n'ait duré qu'un peu de temps. Il a enraciné la tendance lyrique et subjective chez ses poètes, s'est ouvert sur la culture occidentale dont il a traduit la poésie, s'est inspiré de la mythologie, a expérimenté de nouveaux domaines poétiques comme le poème en rime variée (ach-chir al-morsal) et le vers libre. (ABD-ALLAH 2013, 49).

Il est à noter à cet égard que la différence entre la poésie en vers blancs et celle en vers libres est celle d'une poésie métrique non-rimée et une poésie non-métrique non-rimée. Ce dernier est le cas de notre corpus, français et arabe.

\section{Choix du vocabulaire}

Les poètes surréalistes tendent à choisir des mots étranges ou rares. Cela pourrait être expliqué par leur désir de faire jaillir l'imaginaire de leurs lecteurs, ou aussi par un penchant à leur transférer des visions oniriques. Le rêve, le langage, sont les motsclés pour accéder au monde surréaliste.

Leur mécanisme dans le choix des mots bizarres dans leurs poésies, de sorte que ce vocabulaire s'avère comme un délire verbal, n'est pas gratuit. Raphaëlle Herout signale à ce propos que: "non seulement, les mots sont pervertis par ce passé, mais ils sont présentés comme étant la matière première de la pensée : on pense avec les mots dont on dispose, et le problème survient rapidement lorsque l'on se rend compte que les mots, s'ils étaient bons à penser l'époque passée, ne sont plus suffisants pour penser et vivre vraiment l'époque en cours. C'est là le grain de sable dans 
l'engrenage pourtant bien huilé de la reproduction des discours qui apparaît dès les premiers textes surréalistes : l'idée qui ressort dans ces textes est que les mots qui sont disponibles pour ces poètes ne les satisfont pas" (HEROUT 2016).

Ce puisement du vocabulaire illogique vient d'un besoin de montrer la vérité du monde: "les surréalistes vont vouloir renouer avec un langage authentique, expressif, un langage qui ne soit pas un cache-misère, mais au contraire, qui puisse réellement appréhender intensément le réel. Donc les mots, on l'a vu, ne suffisent pas à dire le monde : ils ne suffisent pas non plus à dire le sujet, l'être" (HEROUT 2016).

Le plan des poètes dans leur choix du vocabulaire est aussi précis et clair: "leur entreprise poétique va être de redonner sens aux mots : soit, donner plus de sens, de manière intensive, soit de manière extensive : avoir plus de sens pour dire le monde et donc pour le vivre [...] Pour ne pas rétrécir le grand réel, il y a donc deux options, soit inventer de nouveaux mots [qu'on ne trouve pas dans le Larousse], ce qui est relativement peu fait dans le corpus surréaliste, soit changer l'usage que l'on fait du langage. C'est là je pense une des originalités du surréalisme: tous ces écarts linguistiques que l'on connaît, les détours vers l'impossible de langue sont créés et donnés à lire dans le but d'opérer un renouvellement des usages langagiers pour libérer non pas les mots, mais le sujet parlant." (HEROUT 2016).

Dans Liberté d'Eluard, le poète fait référence au monde extérieur, puisque les quatre éléments y sont présents: l'air, la terre, l'eau et le feu: 
L'air, nous le trouvons présent dans les "chiffons d'azur", dans "l'horizon", dans "les ailes des oiseaux", "le moulin des ombres", dans "chaque bouffée d'aurore" ou dans "les sueurs de l'orage".

La terre se voit dans "les arbres", "la jungle et le désert", "les genêts", "les champs", "la montagne démente", "les sentiers éveillés", "les routes déployées", "les refuges détruits" et les "phares écroulés".

L'eau se trouve dans "la neige", "l'étang", "le lac", "la mer", "les bateaux", et "la mousse des nuages".

Le feu se montre dans le "cendre", "l'étang soleil", "la lampe qui s'allume" et dans "le flot du feu béni". A tout cela s'ajoute un nombre d'éléments avec leur vocabulaire expressif: éléments corporels comme "oreilles", "front", "main", "lèvres"; éléments concrets: "armes", "couronne", "miroir", lit"; éléments abstraits: "vérité", "silence", "ennui", "solitude", "mort", espoir" et "liberté". Ce poème est tellement riche de sens, sons, formes et de couleurs. (VIOUX 2021).

Dans L'ancienne terre de Matar, la présence des quatre éléments de la nature est obsédante. Chaouqi Bazih ${ }^{3}$ parle de la poésie de Matar en disant que "Chaque lecture critique approfondie de l'expérience de Mohamed Afifi Matar ne se redresse sans mettre en considération qu'il est l'un des poètes rares qui voient en la poésie un projet cognitif et visionnaire, et non pas seulement un esthétisme ou un chant de la solitude de l'être humain. Quant à sa tendance de réunir la poésie et la philosophie, c'est plutôt dû à son étude et son enseignement de la philosophie pendant deux décennies. C'est dont on retrace l'impact dans son recueil Aspects du visage d'Empédocle ${ }^{4}$; lorsque l'influence du philosophe grec ne se restreint à l'influence des quatre éléments de 
la création, mais à l'affirmation de la cohérence et la différence, l'amour et la haine, qui sont en conflit permanent dans l'âme humaine". (BAZIH 2020).

La même obsession des quatre éléments de la nature nous la témoignons dans le poème d'Eluard La victoire de Guernica: "Le ciel la terre l'eau le sommeil". (ELUARD 2021).

Le mot "feu" est remplacé dans le vers présent par "sommeil"; une substitution qui désignerait la mort. Dans un vers précédent le mot feu apparait: "Visages bons au feu visages bons au fond". (ELUARD 2021).

Le même thème est fréquent dans Cette nuit de Matar:

"فبلادك انعصفت وسيق هواؤها وترابها سبيًا" (MATAR 2011)

"ton pays a été ravagé, son air et son sol ont été capturés".

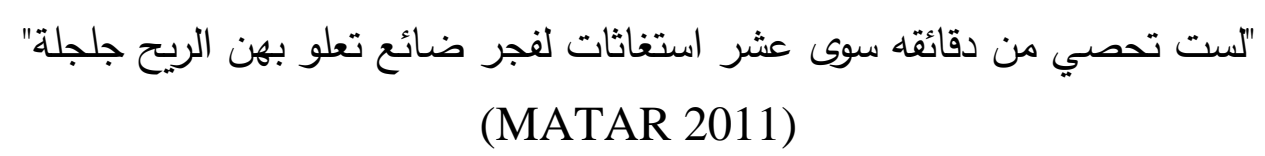

"tu ne comptes de ses minutes que dix cris au secours portés au vent en haut, d'une aurore perdue".

On rencontre parfois des mots qui sont rarement utilisés; l'écriture surréaliste entame des aventures linguistiques à chaque écriture. On ne s'attend pas à lire des mots habituels ou des structures domestiquées. Chez Eluard dans Liberté, un "étang soleil moisi" n'est pas courant dans la poésie, "saisons fiancées" n'est pas familier dans l'usage courant, puisque chez Matar, nous 
trouvons des mots arabes familiarisés par une sorte de résurrection linguistique: le poète les exhume du dictionnaire et les ravive en leur attribuant une présence dans la poésie. Dans L'ancienne terre, nous lisons "السياف طرة"; le front de l'épéiste. Le mot torra n'est plus utilisé. On le remplace dans l'usage courant par gabha. " أنتظرُ "و الأوامرَ الميمونة الطغر اءً bon augure, lorsque le mot togra'a est rarement employés. "شالاًها المنقوطَ المهدودبَ", et son châle à frange et à pois". L'adjectif mohdaoudeb n'est pas fréquent en arabe courant.

Dans son poème Cette nuit, le poète continue assidûment à nous choquer de ces emplois incommuns des mots: " يا -و أنت مجندل آخر الأسرى", "et toi, le dernier captif jeté à terre". Le mot mojandal n'est trouvé que dans le dictionnaire, lorsque son alternative en arabe courant est l'adjectif "صريع". On trouve aussi " فابدأ مقام الكثف للار هبوت", "commence à détecter la terreur". Le mot rahabout n'est pas connu sous cette forme, mais il est connu sous la forme de "رهبة rahba. La forme utilisée par le poète donne un sens hyperbolique et n'existe vraiment que dans le dictionnaire.

"Il est important de noter que le langage - selon Afifi - a commencé à abandonner sa mission originelle de (communication) qu'il exerce dans les discours familiers en général, et non poétiques en particulier, en passant d'un médiateur à une cible en soi." (ABDEL-MOTTALEB 2009).

En cela, les poètes surréalistes tendent à utiliser la langue autrement. Leur moyen est d'extraire des mots rares, ou même de faire des structures bizarres en réunissant des mots courants dont le résultat sera des sens non-accoutumés. Nous verrons dans les 
lignes suivantes quelques emplois de ces structures qui réalisent des figures de styles étonnantes.

\section{Figures de style}

Les figures de style ou de rhétorique, sont les moyens qu'un rhéteur utilisent pour créer un "art de bien parler; [ou la] technique de la mise en œuvre des moyens d'expression." (ROBERT 1993).

Ces moyens, ou techniques, représentent un oblique de langage. Selon Genette, "ce langage oblique qui donne à entendre un sens non proféré, c'est le langage de la connotation, dont la littérature est le domaine par excellence, et dont l'étude peut se prévaloir d'un précédent illustre, quoique décrié: celui de la Rhétorique." (GENETTE, Figures I 1966, 191).

Selon Dominique Combe, " les critiques de la notion des genres, on l'a vu avec Hugo comme avec le surréalisme, visent d'ailleurs en définitive la rhétorique en général, peut-être parce qu'il est plus difficile de s'en prendre aux figures, indispensables à la poésie, à la composition, ou même à l'invention avec laquelle l'inspiration a partie liée" (COMBE 1992, 45).

L'objectif de la critique littéraire en général, c'est de contribuer "à déchiffrer et à constituer de l'intelligible, puisqu'elle est en même temps sémantique et sémantème, sujet et objet de l'activité sémiologique." (GENETTE, Figures I 1966, 188).

Olivier Reboul, préférant quand même la définition classique de la rhétorique, ne s'attarde pas a nous montrer la différence majeure entre deux écoles dans leur définition de la rhétorique: "au début des années soixante, les universitaires ont pourtant retrouvé la rhétorique et ont rendu au mot sa noblesse, à 
la fois prestigieuse et dangereuse; mais sans pour autant se mettre d'accord sur son sens [on en distingue deux positions extrêmes]. L'une [...] voit dans la rhétorique l'art d'argumenter [...]. L'autre [...] font de la rhétorique l'étude du style, et plus particulièrement des figures. Pour les premiers la rhétorique vise à convaincre, pour les seconds, elle constitue ce qui rend un texte littéraire" (REBOUL 1991, 3-4).

Au surplus, "Si l'œuvre est un langage et la critique un métalangage, leur rapport est essentiellement formel, et la critique n'a plus affaire à un message, mais à un code, c'est-à-dire un système dont elle doit retrouver la structure." (GENETTE, Figures I 1966, 187).

Dans la poésie surréaliste, le choix des mots est si expressif au point qu'il semble axial en termes de rhétorique. Le lyrisme traditionnel de la poésie n'a plus de place ici. L'imaginaire ne prend pas pour référent la réalité extérieure; parce que cette réalité se déforme afin de représenter seulement la vision du poète, son inconscient et son rêve.

L'image pour les surréalistes occupe une place privilégiée. Berranger, à ce propos, constate que "l'image n'est donc pas fondée sur une ressemblance entre des objets, mais sur une ressemblance de rapports [...] L'effet stupéfiant qui correspond à l'aspect non prémédité de la rencontre de mots fait de l'image une hallucination subie, créant accoutumance et dépendance." (BERRANGER 1997, 127).

Dans La victoire de Guernica, le poète tend dans le titre du poème à employer l'antiphrase. Cette dernière est une figure 
d'opposition qui exprime une idée par son contraire dans une intention ironique. La seule déviation dans son emploi est que le poète l'a utilisée pour désigner le refus de l'idée de la défaite et la destruction de Guernica par les nazis. Ce refus a été traduit par l'emploi de l'antiphrase, mais en changeant son but. Ainsi le poète fait-il dans le reste de son poème: nous trouvons aussi qu'il a fait fonctionner une autre figure d'opposition, le paradoxe: "Beau monde des masure/ De la nuit et des champs". Le paradoxe est une "opinion qui va à l'encontre de l'opinion communément admise." (ROBERT 1993). Cette figure de style consiste à exprimer une opinion contraire à l'idée commune, afin de surprendre, de choquer ou d'inviter à la réflexion. Donc, quelle sorte de beauté pourrons-nous voir dans les masures? Ce type de figure d'opposition n'est pas uniquement présent dans la poésie surréaliste. Pour les poètes, il leur tient à cœur de multiplier les antithèses, les paradoxes, les oxymores et les antiphrases. Cela reflète amplement leur refus du réel avec toute sa contradiction, sa subversion et son désordre. L'antithèse, qui consiste à opposer fortement deux termes, est significative à cet égard: "Votre mort va servir d'exemple", "Et la misère/ De votre vie"; "Les femmes les enfants ont le même trésor/ Dans les yeux/ Les hommes le défendent comme ils peuvent"; "La peur et le courage de vivre et de mourirl La mort si difficile et si facile"; "Hommes réels pour qui le désespoir/ Alimente le feu dévorant de l'espoir".

La paronomase, comme une figure de sonorité, est pareillement utilisée: cette figure qui se compose d'un rapprochement de deux homonymes (qui se prononcent pareil) ou de deux paronymes (qui se prononcent presque pareil), afin de produire un effet musical ainsi qu'un effet mental: "Visages bons au feu visages bons au fond". 
Les figures de sonorité sont à la base de la poésie surréaliste qui passe sans rime ni mètre ou rythme régulier. Donc, nous dégageons plein de figures comme l'allitération et l'assonance. L'allitération est une "répétition des consonnes initiales (par extension: des consonnes intérieures) dans une suite de mots rapprochés" (ROBERT 1993): "Visages bons à tout/ Voici le vide qui vous fixe/ Votre mort va servir d'exemple". Dans les vers précédents, le son " $v$ " est fréquent; ce qui fait un effet de vibration ou d'agitation propres à l'atmosphère de la guerre. On trouve évidemment aussi cette rhétorique dans les vers suivants: "Ils disaient désirer la bonne intelligence/ Ils rationnaient les forts jugeaient les fous/ Faisaient l'aumône partageaient un sou en deux/ Ils saluaient les cadavres/ Ils s'accablaient de politesses". Les sons "d", "z" et "s" sont respectivement fréquents. Le tapement et le sifflement de ces consonnes contribuent à l'atmosphère rigide et tendu du poème parlant de la mort qui est partout.

L'assonance est une "répétition du même son de la voyelle accentuée à la fin de chaque vers" (ROBERT 1993). Pour la distinguer de la rime, l'assonance s'intéresse aux sons vocaliques de l'ensemble des vers ou des strophes, ainsi que la rime s'intéresse uniquement du son de la fin de chaque vers. Nous la trouvons fréquente dans les vers: le son "i" est tant employé: "Visages bons à tout/ Voici le vide qui vous fixe/Votre mort va servir d'exemple". Nous l'avons aussi dans le vers suivant: "Ils disaient désirer la bonne intelligence". Cette répétition reflète l'acuité des cris et des tristesses qu'entrainent la guerre et la douleur.

La nasalité est omniprésente dans le poème: le son "an" se trouve dans "champs; exemple; enfants; défendent; sang; chanté"; 
lorsque le son "on" s'entend dans des mots comme: "monde; bon; fond; sont; ont"; ce qui nous transmet le gémissement et la peine d'un peuple qui a tant souffert des répercussions de la guerre.

Comme nous l'avons signalé supra, la musique des vers ne se produit pas de la rime ni du mètre, mais seulement de la musicalité intérieure du poème, créée par une abondance des effets vocaux. L'anaphore est parmi ces techniques musicales tant employées: cette figure d'insistance est une "répétition d'un mot en tête de plusieurs membres de phrases, pour obtenir un effet de renforcement ou de symétrie." (ROBERT 1993). C'est aussi la reprise de mêmes termes en début de plusieurs vers pour marteler une idée ou y insister et souligner. Cette figure produit également une musique pareille à celle des chants: dans des vers suivis aux différentes séquences, on rencontre par exemple: "Les femmes les enfants ont le même trésor", "Les femmes les enfants ont les mêmes roses rouges "; "Hommes pour qui ce trésor fut chanté/ Hommes pour qui ce trésor fut gâché".

Il y a aussi maints exemples de figures syntaxiques de rupture dans la poésie surréaliste: nous rencontrons beaucoup d'ellipses et d'anacoluthes. Cette figure dernière est parfois considérée comme conçue à l'ellipse. Elle est une sorte d'ellipse par laquelle on omet dans une phrase le mot, le terme qui est le corrélatif ordinaire de l'un des mots, des termes exprimés. Selon le dictionnaire, elle est une "rupture ou discontinuité dans la construction d'une phrase" (ROBERT 1993). La poésie surréaliste en abonde: "La mort cœur renversé"; c'est-à-dire la mort est un cœur renversé. Cette rupture est dans l'intention du raccourci. 
Ajoutons aux figures de rupture le zeugma, ou l'attelage: il consiste à faire un rapprochement d'un mot concret et d'un mot abstrait dans un même énoncé d'une manière faisant cette rupture mentale et logique: "Hommes réels pour qui le désespoir/ Alimente le feu dévorant de l'espoir".

On trouve aussi l'hypallage, cette figure de style consistant à "attribuer à certains mots d'une phrase ce qui convient à d'autres mots" (ROBERT 1993). Ce déplacement se réalise sans que cela empêche de comprendre le sens. L'exemple suivant le représente clairement: "Ouvrons ensemble le dernier bourgeon de l'avenir".

La gradation et l'accumulation sont des figure d'insistance: l'accumulation est une énumération plus ou moins longue de termes, dans un but d'amplification ou d'excès.: "Visages bons au feu visages bons au fond/ Aux refus à la nuit aux injures aux coups". Dans une autre séquence, on lit: "Ils vous ont fait payer le pain/ Le ciel la terre l'eau le sommeil/ Et la misère/ De votre vie". Puisque la gradation est une énumération de termes, organisée de façon croissante ou décroissante: "Ils disaient désirer la bonne intelligence/ Ils rationnaient les forts jugeaient les fous/ Faisaient l'aumône partageaient un sou en deux/ Ils saluaient les cadavres/ Ils s'accablaient de politesses". Nous avons aussi: "Ils persévèrent ils exagèrent ils ne sont pas de notre monde"; et "Les femmes les enfants ont le même trésor/ De feuilles vertes de printemps et de lait pur/ Et de durée/ Dans leurs yeux purs".

Berranger pose une question sur la liberté en tant qu'objectif chez les surréalistes: "La liberté serait-elle la finalité de la morale surréaliste? C'est bien le même mot qui signale l'effet de l'une ou de l'autre: l'exaltation. Amour, morale, liberté sont une trinité: 
aucune de ces valeurs n'est indépendante des autres. La liberté politique en dernier ressort apparait cependant comme le moyen de disposer enfin de soi, et de vivre sans frein la passion". (BERRANGER 1997, 76)

Cette dernière notion sur la liberté politique est un principe provocateur des surréalistes: pour qu'on pratiquer la liberté de l'écriture sans contraintes sociales ou langagières, il faut défendre leur liberté politique.

Liberté, ce poème porte en lui un panache d'images poétique et de figures de rhétorique surprenant: le poème se caractérise par les débuts anaphoriques: chaque vers dans chaque strophe, sauf le refrain, commence par le mot "sur": ex. "Sur mes cahiers d'écolier/ Sur mon pupitre et les arbres/ Sur le sable sur la neige/ J'écris ton nom" (vs. 1-4) (ELUARD, Liberté 2012). On a appris que l'anaphore est l'une des figures d'insistance, et dont l'emploi produit un effet musical pareil à celui des chansons.

L'accumulation anime le poème. Cette figure d'insistance, et par l'absence de la ponctuation, produit un effet d'abondance convenable a l'ambiance cosmique: "Pierre sang papier ou cendre" (v. 7).

Tout le poème semble suivre un plan amplifié de gradation progressive en montrant le cycle de la vie: dans les quatre premières strophes, on évoque le monde de l'enfance: "cahiers d'écolier; pupitre" (v. 12); "l'écho de mon enfance". (v. 15). On passe par la jeunesse en évoquant "saisons fiancées" (v. 19); "chair accordée" (v. 61), "lèvres attentives" (v. 66). Ces strophes sont suivies des autres parlant de la vieillesse avec le vocabulaire 
qui la représente: "la solitude nue" (v. 74); "les marches de la mort" (v. 75). (VIOUX 2021)

L'allitération est répandue dans tout le poème: on entend une abondance des sons "l", "p", "r" et "s"; ce qui reflète cet encombrement de sons issus de divers aspects cosmiques et naturels.

L'assonance est aussi présente; le son vocalique "é" est partout: "Sur les merveilles des nuits/ Sur le pain blanc des journées/ Sur les saisons fiancées/ J'écris ton nom" (vs. 17- 20).

La paronomase redouble la sonorité et la musicalité des poèmes: "Pierre sang papier ou cendre" (v. 7). Le son "i" n'est pas moins commun: "Sur la vitre des surprises/ Sur les lèvres attentives/ Bien au-dessus du silence/ J'écris ton nom" (vs. 65$68)$.

L'antithèse est aussi omniprésente: cette figure d'opposition est très chère aux poètes surréalistes qui y trouvent le reflet de leur vision du monde: "Sur la jungle et le désert" (v.13); "Sur les merveilles des nuits/ Sur le pain blanc des journées" (vs. 17- 18); "soleil moisi/lune vivante" (vs. 22- 23); "Sur la lampe qui s'allume/ Sur la lampe qui s'éteint" (vs. 45- 46); "la santé revenue/ le risque disparu" (vs. 77- 78).

Et comme cette manie de l'opposition harcèle le poète, nous voyons que l'oxymore trouve sa place dans son poème: "Sur le flot du feu béni" (v.59). Cette dernière figure consiste à "allier deux mots de sens contradictoires pour leur donner plus de force expressive" (ROBERT 1993). C'est donc une alliance surprenante de mots opposés qui renforce une idée. 
Le paradoxe, parmi les figures d'opposition, s'exprime dans le poème à travers ce vers: " Sur le pain blanc ${ }^{5}$ des journées" (v $18)$.

Les figures de substitution sont multiples dans le poème; on en compte aussi la synecdoque: elle, comme un cas particulier de la métonymie, consiste à designer la partie pour le tout, ou vice versa. L'exemple suivant de Liberté l'explique: "Sur les lèvres attentives" (v. 66).

Le poète multiplie l'emploi de l'hypallage dans son poème: la fonctionnalisation de cette figure traduit le goût surréaliste du bizarre et de l'inaccoutumé: "Sur les saisons fiancées" (v.19); " Sur l'étang soleil moisi/ Sur le lac lune vivante" (vs. 22- 23); "la montagne démente" (v. 31);" les cloches des couleurs" (v. 38); "les sentiers éveillés" (v. 41); "la vitre des surprises" (v. 65); "les murs de mon ennui" (v. 71); "la solitude nue" (v. 74). Il est à signaler que tous les exemples précédents sont de l'hypallage in absentia; ce type, au contraire de l'hypallage in praesentia, n'exige pas la présence des termes dont on déplace les adjectifs. Cette figure donne un effet de personnification, puisqu'elle renvoie un attribut humain à une vérité non-humaine; donc, on peut la classer soit en tant que figure de substitution soit une figure d'analogie.

La personnification, comme une figure d'analogie a part, représente une idée ou une chose sous les traits d'une personne: "Sur les marches de la mort" (v. 75). Donc, la mort est personnifiée en tant qu'un être humain qui avance avec des marches irréversibles. 
La strophe finale semble porter la réponse d'une question posée dès le début: qu'est-ce que c'est le mot magique que chante le poète tout au long du poème, et qu'il écrit sur tous les aspects du cosmos?: " Et par le pouvoir d'un mot/ Je recommence ma vie/ Je suis né pour te connaître/ Pour te nommer/ Liberté" (vs.82- 86). Cette strophe incantatoire, nous donne la clé, le mot magique chanté dans tous les temps, la liberté.

Dans le tableau récapitulatif suivant nous montrons la diversité rhétorique que le poete avait employée dans ses deux poèmes:

\begin{tabular}{|c|c|c|c|c|c|c|c|c|c|c|c|c|c|c|c|c|}
\hline \multicolumn{4}{|c|}{$\begin{array}{c}\text { Figures } \\
\text { d'opposition }\end{array}$} & \multicolumn{3}{|c|}{$\begin{array}{c}\text { Figures } \\
\text { d'insistanc } \\
\text { e }\end{array}$} & \multicolumn{3}{|c|}{$\begin{array}{l}\text { Figures de } \\
\text { rupture } \\
\text { grammatic } \\
\text { ale }\end{array}$} & \multicolumn{3}{|c|}{$\begin{array}{l}\text { Figures de } \\
\text { sonorité }\end{array}$} & \multicolumn{3}{|c|}{$\begin{array}{c}\text { Figures de } \\
\text { substitutio } \\
\text { n }\end{array}$} & \multirow[t]{2}{*}{$\begin{array}{c}\text { Figures } \\
\text { d'analo } \\
\text { gie }\end{array}$} \\
\hline $\mathrm{A}$ & $\mathrm{P}$ & $\mathrm{A}$ & $\mathrm{O}$ & $\mathrm{A}$ & $\mathrm{A}$ & $\mathrm{G}$ & $\mathrm{A}$ & $\mathrm{Z}$ & $\mathrm{H}$ & $\mathrm{A}$ & $\mathrm{A}$ & $\mathrm{P}$ & $S$ & $\mathrm{H}$ & $\mathrm{P}$ & \\
\hline $\mathrm{n}$ & $\mathrm{a}$ & $\mathrm{n}$ & $\mathrm{x}$ & $\mathrm{n}$ & $\mathrm{c}$ & $\mathrm{r}$ & $\mathrm{n}$ & $\mathrm{e}$ & $\mathrm{y}$ & 1 & $\mathrm{~s}$ & $\mathrm{a}$ & $\mathrm{y}$ & $\mathrm{y}$ & é & Perso \\
\hline $\mathrm{t}$ & r & $\mathrm{t}$ & $\mathrm{y}$ & $\mathrm{a}$ & $\mathrm{c}$ & $\mathrm{a}$ & $\mathrm{a}$ & $\mathrm{u}$ & $\mathrm{p}$ & 1 & $\mathrm{~s}$ & $\mathrm{r}$ & $\mathrm{n}$ & $\mathrm{p}$ & $\mathrm{r}$ & nnific \\
\hline i & $\mathrm{a}$ & i & $\mathrm{m}$ & $\mathrm{p}$ & $\mathrm{u}$ & d & $\mathrm{c}$ & $\mathrm{g}$ & $\mathrm{a}$ & i & o & o & $\mathrm{e}$ & $\mathrm{a}$ & $\mathrm{i}$ & ation \\
\hline $\mathrm{p}$ & $\mathrm{d}$ & $\mathrm{t}$ & o & $\mathrm{h}$ & $\mathrm{m}$ & $\mathrm{a}$ & o & $\mathrm{m}$ & 1 & $\mathrm{t}$ & $\mathrm{n}$ & $\mathrm{n}$ & $\mathrm{c}$ & 1 & $\mathrm{p}$ & \\
\hline h & o & $\mathrm{h}$ & $\mathrm{r}$ & o & $\mathrm{u}$ & $\mathrm{t}$ & 1 & $\mathrm{a}$ & 1 & é & $\mathrm{a}$ & o & d & 1 & h & \\
\hline$r$ & $\mathrm{x}$ & è & $\mathrm{e}$ & $r$ & 1 & i & $\mathrm{u}$ & & $\mathrm{a}$ & $r$ & $\mathrm{n}$ & $\mathrm{m}$ & o & $\mathrm{a}$ & r & \\
\hline $\mathrm{a}$ & $\mathrm{e}$ & $\mathrm{s}$ & & $\mathrm{e}$ & $\mathrm{a}$ & o & $\mathrm{t}$ & & $\mathrm{g}$ & $\mathrm{a}$ & $\mathrm{c}$ & $\mathrm{a}$ & $\mathrm{q}$ & $\mathrm{g}$ & $\mathrm{a}$ & \\
\hline s & & $\mathrm{e}$ & & & $\mathrm{t}$ & $\mathrm{n}$ & h & & $\mathrm{e}$ & $\mathrm{t}$ & $\mathrm{e}$ & $\mathrm{s}$ & $\mathrm{u}$ & $\mathrm{e}$ & $\mathrm{s}$ & \\
\hline e & & & & & i & & $\mathrm{e}$ & & & i & & $\mathrm{e}$ & $\mathrm{e}$ & & e & \\
\hline & & & & & $\begin{array}{l}\mathrm{o} \\
\mathrm{n}\end{array}$ & & & & & $\begin{array}{l}\mathrm{o} \\
\mathrm{n}\end{array}$ & & & & & & \\
\hline
\end{tabular}


Chez Matar, la rhétorique pourrait se différencier de la rhétorique française dans quelques points; mais les deux se rapprochent dans d'autres.

Le poème intitule L'ancienne terre s'avère d'une ambiguïté extrême: le vocabulaire utilisé est rétabli comme si les mots n'appartiennent les uns aux l'autres. Si on a de la chance pour comprendre un peu les poèmes d'Eluard c'est parce que l'occasion pour laquelle le poète avait créé ces poèmes est connue. Le cas n'est pas pareil pour Matar dont l'écriture subit une expérience générale de refus et de résistance contre l'injustice.

La prison est une partie importante de cette expérience poétique aussi que la révolte contre les régimes politiques. Le poème avait été écrit après la signature des accords de Camp David $^{6}$ en 1979 entre l'Egypte et Israël, contestée de la part des cultivés en particulier et du peuple égyptien en général. Ceux-ci y trouvent une humiliation qui ne convient pas avec le statut du vainqueur, qui est l'Egypte, après sa victoire dans la guerre de 1973.

Matar, souffrant comme les Egyptiens et les Arabes des retombées politiques de ces accords, avait écrit ce poème militant. Le choix des mots cruels et forts reflète cette douleur.

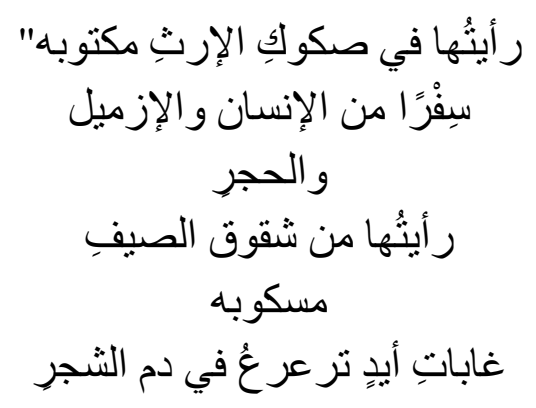




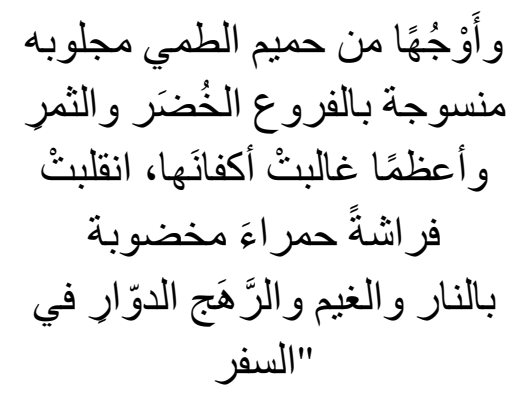

(MATAR 2011)

Je l'ai vue écrite dans les actes de succession comme un livre d'homme, de ciseau et de pierre

Je l'ai vue depuis les fissures de l'été, déversée

Des forêts de mains qui poussent dans le sang des arbres

Et des visages du limon chaud sont amenés

Tissés avec des branches vertes et des fruits

Et des os entassés dans ses linceuls, tous transformés en un papillon rouge fardé

Avec du feu, des nuages et des éclairs tourbillonnants au voyage

Cette chose inconnue, que le poète voit à travers divers aspects de la nature, nous ne pouvons pas la deviner sans lier le poème, dans son entité, avec l'occasion pour laquelle il est écrit. Ce qui pourrait être écrit dans les actes de succession, ce sont les possessions transférées des ancêtres aux descendants, qui sont les héritiers. Si le patrimoine est un livre d'homme, de ciseau et de pierre, donc ce seront les outils indispensables pour rétablir une civilisation. Cette dernière on la tâtonne dans la canicule au temps du labeur, dans les mains blessées à cause du travail dans la terre, dans les visages bruns, marqués par la couleur du limon. 
L'Homme crée la civilisation: il produit la verdure et les fruits de la terre; après sa mort ses os se mêlent avec la terre; son âme se transforme en un papillon rouge, une liberté tachetée de la couleur du sang, parce que la liberté n'est pas gratuite.

Le fait de prétendre déchiffrer un texte poétique de Matar ressemble au fait de résoudre une énigme insoluble. Mais, on peut démarrer par des pistes qui peuvent nous aider à avoir des clés pour comprendre: "dans une interview avec lui, il est accusé d'ambiguïté dans sa poésie, il a répondu à ce point en disant qu'entre moi et ma poésie il n'y a pas d'ambiguïté, mais je ne ressens son ambiguïté, sa fermeture et sa complexité que lorsque on me pose des questions de la part de certains critiques [sur le sens de ces poèmes]" (Al-MAGRAHI 2021).

Le début anaphorique de "je l'ai vue" qui se répète dans le premier et le quatrième vers, fait cet écho musical agréable à l'oreille.

L'accumulation dans le vers suivant "un livre d'homme de ciseau/ et de pierre" met en valeur les moyens par lesquels la civilisation se rétablit.

l'hypallage in absentia est tant utilisée par les surréalistes: dans le vers suivant: " les fissures de l'été", nous touchons l'attribut d'un mot à ce qui convient à un autre sans qu'on perde la compréhension du contexte.

Une autre hypallage se trouve dans les vers suivants: "un papillon rouge fardé/ Avec du feu, des nuages et des éclairs tourbillonnants". 
La métaphore comme une figure d'analogie, est la figure de style la plus chère aux poètes modernes, surtout les surréalistes. A ce propos, Genette dit: "Il me semble en effet que le profond désir de toute une poétique moderne est bien à la fois de supprimer les partages et d'établir le règne absolu - sans partage- de la métaphore. Le reste n'est peut-être que motivation. Le mouvement séculaire de réduction de la rhétorique semble donc aboutir à une valorisation absolue de la métaphore." (G. GENETTE 1972, 49).

Le débat sur la valeur sémantique de la métaphore dans le discours littéraire s'accentue par la parole de Kristeva, commentant Breton: "le poète n'a pas voulu dire autre chose qu'il ne dit, mais les mots disent dans les métaphores autre chose qu'ils ne signifient habituellement." (TODOROV 1979).

La métaphore se présente dans le poème de Matar dans plusieurs endroit: "Des forêts de mains qui poussent dans le sang des arbres". Dans cette image, nous avons une assimilation entre un comparé et un comparant sans la présence d'un terme de comparaison. On compare les mains a des forêts. Donc, le comparé est "mains", tandis que le comparant est "forêts".

On a aussi une autre métaphore dans ce vers: "Et des visages du limon chaud sont amenés/ Tissés avec des branches vertes et des fruits": les visages du limon sont ici comparés à une étoffe tissée avec des branches et des fruits.

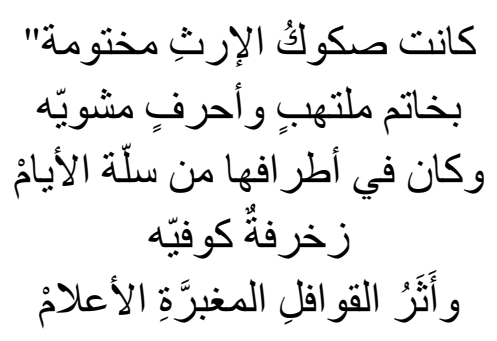




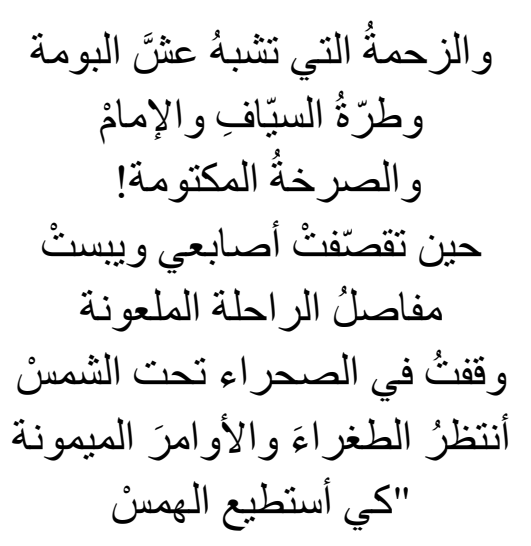

(MATAR 2011)

Les actes de succession ont été scellés

Avec un sceau enflammé et des lettres grillées

Et il y avait sur ses bords du panier des jours

une décoration coufique

Et des traces de caravanes aux drapeaux poussiéreux

Et un embouteillage ressemblant au nid de chouette

Et le front de l'épéiste et de l'imam

Et le cri étouffé !

Quand mes doigts sont cassés

Et les articulations de la bête maudite ont été séchées

Je me tenais dans le désert sous le soleil

En attendant le monogramme et les ordres de bon augure

Pour que je puisse chuchoter.

Les vers précédents montrent l'influence de la culture arabe islamique sur le poète: des termes comme "coufique; caravanes, épéiste; imam" prouvent cet impact. Le patrimoine égyptien est un mélange de plusieurs civilisations entrelacées et harmonieuses. Cette phase ferait allusion à la conquête islamique de l'Egypte. ${ }^{7}$ Une période qui avait tant formé l'identité égyptienne qui se moule selon la culture dominante. 
L'hypallage existe avec vigueur: les poètes surréalistes la privilégient aux autres figures de style, parce qu'elle exprime leur vision envers le monde: "un sceau enflammé et des lettres grillées". Dans ce même vers, nous touchons la gradation dans ce décalage entre deux cas de brûlure: "enflammé" et "grillées".

La périphrase comme une figure de substitution consiste à "exprimer une notion qu'un seul mot pourrait designer par un groupe de plusieurs mots" (ROBERT 1993). On remplace un mot par une expression qui le définit. Un simple mot est remplacé par des éléments de phrase plus complexes, jouant sur l'implicite. Le "panier des jours" désigne le temps. L'image ici est composée: une hypallage et une périphrase.

La comparaison, dont le comparé, le comparant et l'outil de comparaison existe dans ce vers: "Et un embouteillage ressemblant au nid de chouette". Si le lien entre le comparé et le comparant n'est pas clair dans l'exemple présent, c'est parce que la vision du poète surréaliste n'est pas concevable.

L'oxymore est aussi une figure très chère aux poètes surréalistes: "le cri étouffé"; deux mots contradictoires unis.

$$
\begin{aligned}
& \text { وقفتُ في الصحر اءء و اجمًا مُقنَّعًا }
\end{aligned}
$$

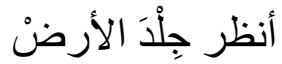

$$
\begin{aligned}
& \text { مستبدلًا منتسخًا مرقَّعَا } \\
& \text { وكلما ثبتتبت الثمسُ رماحَها } \\
& \text { الحمر اءَ في جمجمني }
\end{aligned}
$$

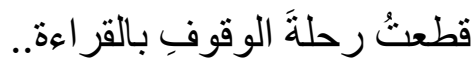

$$
\begin{aligned}
& \text { أُخرج من غيابة العباءة } \\
& \text { صحائفت الإرثِ المقدسة } \\
& \text { وكلما تخرّقتْْ سطورُ ها تها }
\end{aligned}
$$




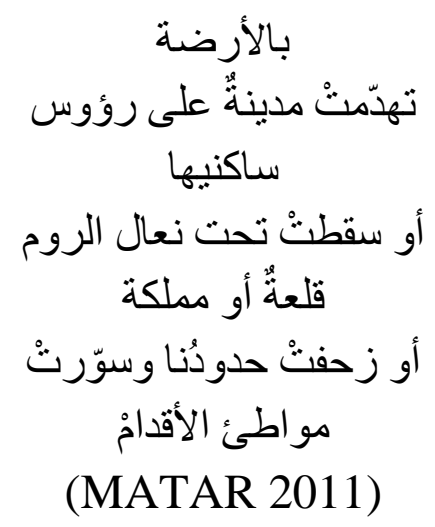

Je me tenais dans le désert, silencieux morose masqué, voir la peau de la terre, changé, cloné, rapiécé

Et quand le soleil a fixé ses lances rouges dans mon crâne J'ai interrompu le voyage d'attente par la lecture je fais sortir du fond du manteau les actes de succession sacrés

Et quand ses lignes sont percées, par le termite Une ville s'est effondrée sur les têtes de ses habitants

Ou est tombé sous les chaussons des Romains un château ou un royaume Ou nos frontières sont prises, clôturées et écrasées

Les actes de successions sembleraient renvoyer aux traités de paix entre l'Egypte et Israël; ce qui montre qu'il s'agissait de lettres mortes parce que les villes s'effondrent sur les têtes de leurs habitants et les frontières sont encore prises par les occupants. 
L'accumulation se trouve dans le vers suivant: "Je me tenais dans le désert, silencieux morose masqué". Dans le texte original la ponctuation n'existe pas, mais la traduction des vers nous exige de la respecter pour donner une approche du sens voulu. Nous avons aussi le mot arabe ouaa'jeman, un seul mot traduit en deux: "silencieux morose". Le silence, assamtu, ou assokoun, n'égale pas al-oujoum (الوجوم), le silence avec tristesse, avec réflexion profonde. Ce vers se parfait par la gradation, ou plutôt la dégradation, dans trois cas de modification psychique subis par le poète: "changé, cloné, rapiécé".

La personnification est amplement employée chez Matar: "voir la peau de la terre". La même figure dans le vers: "quand le soleil a fixé ses lances". Le soleil est comparé à un guerrier qui dirige ses lances vers son ennemi.

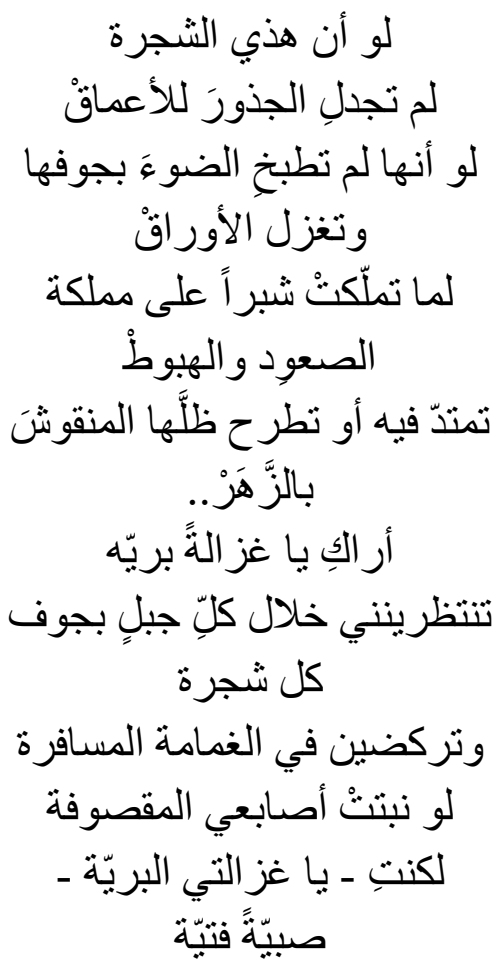




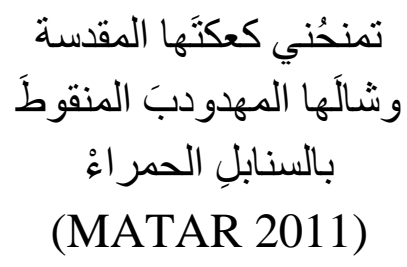

Si cet arbre

N'avait pas tressé les racines aux profondeurs

S'il ne faisait pas cuire la lumière en lui,

$\mathrm{Ni}$ filait les feuilles

Il ne posséderait jamais un pouce du royaume

Ascendant et descendant

Où il étend ou projette son ombre gravée

Avec des fleurs..

Je te vois, cerf sauvage

M'attendant à travers chaque montagne au creux

De chaque arbre

Et tu cours dans les nuages qui voyagent

Si mes doigts coupés grandissaient

Tu serais - mon cerf sauvage -

une jeune fille

Qui me donne son saint gâteau

Et son châle à franges, tacheté

Avec des sépales rouges

La personnification est évidente dans les trois vers suivis: "Si cet arbre/ N'avait pas tressé les racines aux profondeurs/ S'il ne faisait pas cuire la lumière en lui,/ $\mathrm{Ni}$ filait les feuilles". L'arbre/femme tresse ses cheveux (racines), fait cuire la nourriture (lumière), et file avec une quenouille les étoffes de ses vêtements (feuilles). 
"Je te vois, cerf sauvage": cet animal doux est aussi personnifié avec un tutoiement intime quand le poète s'adresse à lui.

L'antithèse est aussi employée: "Ascendant et descendant". Cette opposition accentue la valeur des mots.

L'hyperbole, comme une figure d'insistance, consiste à exagérer, à "mettre en relief une idée au moyen d'une expression qui la dépasse." (ROBERT 1993). Elle donne du relief pour mettre en valeur une idée, un sentiment. On la rencontre dans ce vers: "Si mes doigts coupés grandissaient".

Quant aux figures de sonorité, il n'est pas question de les discuter ici à cause de la traduction. Les mots traduits perdent par conséquent leur caractère musical d'une langue à l'autre, et dans notre cas de l'arabe vers le français, à la suite de la perte de leur forme. Ce serait plus convenable si on les explique dans leur langue d'origine. Ce qui n'est pas notre problématique dans la recherche présente.

Le deuxième poème de Afifi Matar est Cette nuit. Ce poème avait été écrit lors d'une détention à la geôle de Tora en 1991 à cause de ses opinions contestataires du régime politique.

$$
\begin{aligned}
& \text { هذا الليل يبدأ } \\
& \text { دهر من الظلمات أم هي ليلة جمعت سو اد لاد }
\end{aligned}
$$

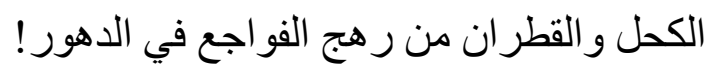

$$
\begin{aligned}
& \text { عينالك تحت عصابة عقدت وساخت في } \\
& \text { عظام الر أس عقدتها، } \\
& \text { وأنت مجندل - يا آخر الأسرى عقدي } \\
& \text { ولست بمفتدى }
\end{aligned}
$$




\section{(MATAR 1991)}

Cette nuit commence

Est-ce un âge de ténèbres, ou une nuit qui a rassemblé la noirceur du Kohl et du goudron au moyen de l'éclair des malheurs dans les

$$
\text { âges ! }
$$

Tes yeux sont sous une bande nouée et enlisée

dans les os de la tête,

Et tu es jeté à terre, toi, le dernier des captifs

tu n'es jamais racheté

Un début anaphorique qui se répète cinq fois dans de différents endroits du poème: "Cette nuit commence".

L'oxymore dans "l'éclair des malheurs", montre l'opposition dans l'union entre deux termes contradictoires.

L'hyperbole se découvre à travers cette image: "Tes yeux sont sous une bande nouée et enlisée/ dans les os de la tête".

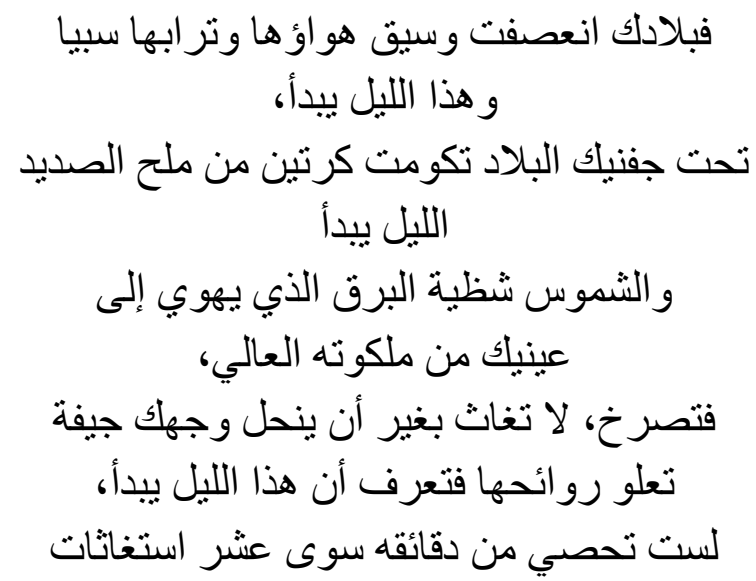




$$
\text { لفجر ضائع تعلو بهن الريح جلجلة }
$$

Ton pays a été ravagé, son air et son sol ont été capturés

Et cette nuit commence

Sous tes paupières le pays s'est amoncelé en deux boules de pus

La nuit commence

Et les soleils sont un éclair qui tombe au-dessous

de tes yeux de son haut royaume,

Alors tu cries, tu ne seras pas sauvé à moins que ton visage ne soit dissous en dépouille

dont les parfums émanent et tu sais que cette nuit commence,

Tu ne peux compter de ses minutes que dix cris au secours

Pour une aube perdue, portés au vent en haut,

Aux horizons des larmes divines

On a une personnification dans l'image: "son air et son sol ont été capturés".

Une hyperbole montrant l'exagération et l'amplification de la vision surréaliste se voit dans le vers suivant: "Sous tes paupières le pays s'est amoncelé en deux boules de pus".

La figure est aussi employée dans ces vers: "les soleils sont un éclair qui tombe au-dessous/ de tes yeux de son haut royaume". C'est redoublé par l'emploi du mot "soleils" en forme du pluriel.

Le contraire de cette figure de style est employé dans la même strophe, ce qui appuie le sens et accentue la valeur poétique des vers: le poète utilise l'euphémisme comme opposant à l'hyperbole. L'euphémisme est une "expression atténuée d'une 
notion dont l'expression directe aurait quelque chose de déplaisant, de choquant." (ROBERT 1993). Le poète en écrivant "tu ne seras pas sauvé à moins que ton visage ne soit dissous en dépouille", ne veut dire que: tu ne seras sauvé a moins que tu sois mort! Il atténue cette vérité austère par l'emploi des termes moins durs.

$$
\begin{aligned}
& \text { هذا الليل يبدأ } \\
& \text { فابتدئ موتا لحلمك و ابتدع حلما لموتلك لبد الجيد } \\
& \text { أيها الجسد الصبور } \\
& \text { الخوف أقسى ما تخاف.. ألم تقل؟! }
\end{aligned}
$$

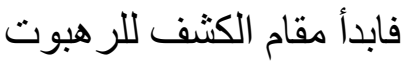

$$
\begin{aligned}
& \text { و انخل من رمادك، و انكثَف عنك، } \\
& \text { اصطف الآفاق مما يبدع الرخ الجسور }
\end{aligned}
$$

Cette nuit commence

Alors commence la mort de ton rêve et invente un rêve pour ta mort toi, corps patient!

La peur est la chose la plus dure que tu crains. N'as-tu pas dit ?! Alors commence à détecter la terreur Et crible tes cendres, et révèles-toi, Alignes-toi aux horizons de ce que crée le $\operatorname{roc}^{8}$ audacieux.

Le début anaphorique se répète encore: "Cette nuit commence".

Il y a un chiasme, cette figure de rhétorique formée d'un croisement des termes." (ROBERT 1993). C'est ainsi une figure d'opposition qui représente deux expressions qui se suivent, dont 
la deuxième adopte l'ordre inverse $(\mathrm{A}-\mathrm{B} / \mathrm{B}$ ' - A'): " Alors commence la mort de ton rêve et invente un rêve pour ta mort ". Cette figure produit un effet musical aimable.

Une autre personnification montre le corps en chair et en os, comme une créature faible mais résistante auquel le poète s'adresse: "Toi, corps patient!". Cette image reflète ce clivage moral du poète, sa division psychique en deux.

L'hyperbole est toujours là pour mettre en relief l'idée du poète: "Et crible tes cendres". Il y a encore ce vers: "Alignes-toi aux horizons de ce que crée le roc audacieux". Il est à noter que le poète est connu par sa passion de l'emprunt des images mythiques. Il utilise le mot "roc" pour affirmer cette obsession.

Somme toute, pour prétendre comprendre Matar, on se doit des compétences distinguées: Ramadan Bastaouissi l'exprime en disant que : "C'est un poète philosophe, éduqué avec une culture large et étendue, et ce n'est pas facile pour nos critiques traditionnels de suivre ses œuvres, de les analyser et d'identifier leurs caractéristiques philosophiques, artistiques et stylistiques les plus importantes. Il a besoin d'un critique qui considère la critique comme une expérience dans laquelle beaucoup d'aventure est impliquée pour traiter le texte avec honnêteté et créer un dialogue correspondant et responsable avec le texte, et non pas le lire à travers des classifications et des préjugés tout faits." (BASTAOUISSI 2009).

Le tableau récapitulatif suivant révèle les figures de rhétorique utilisées de la part de Matar dans ses deux poèmes: 


\begin{tabular}{|c|c|c|c|c|c|c|c|c|c|c|c|}
\hline \multicolumn{3}{|c|}{$\begin{array}{c}\text { Figures } \\
\text { d'opposition }\end{array}$} & \multicolumn{4}{|c|}{$\begin{array}{c}\text { Figures } \\
\text { d'insistance }\end{array}$} & \multicolumn{2}{|c|}{$\begin{array}{l}\text { Figures de } \\
\text { substitution }\end{array}$} & \multicolumn{3}{|c|}{$\begin{array}{c}\text { Figures } \\
\text { d'analogie }\end{array}$} \\
\hline $\mathrm{A}$ & $\mathrm{O}$ & $\mathrm{C}$ & A & $\mathrm{A}$ & $\mathrm{G}$ & $\mathrm{H}$ & $\mathrm{H}$ & Pé & $\mathrm{M}$ & $\mathrm{P}$ & $\mathrm{C}$ \\
\hline $\mathrm{n}$ & $\mathrm{X}$ & $\mathrm{h}$ & $\mathrm{n}$ & $c$ & $r$ & $\mathrm{y}$ & yp & ri & é & $\mathrm{e}$ & $\mathrm{O}$ \\
\hline $\mathrm{t}$ & $\mathrm{y}$ & $\mathrm{i}$ & $\mathrm{a}$ & $c$ & $\mathrm{a}$ & $\mathrm{p}$ & all & $\mathrm{ph}$ & $\mathrm{t}$ & $r$ & $\mathrm{~m}$ \\
\hline $\mathrm{i}$ & $\mathrm{m}$ & $\mathrm{a}$ & $\mathrm{p}$ & $\mathrm{u}$ & d & e & $\mathrm{ag}$ & $\mathrm{ra}$ & $\mathrm{a}$ & $S$ & $\mathrm{p}$ \\
\hline $\mathrm{t}$ & O & $S$ & h & $\mathrm{m}$ & $\mathrm{a}$ & $r$ & e & Se & $\mathrm{p}$ & O & $\mathrm{a}$ \\
\hline $\mathrm{h}$ & $r$ & $\mathrm{~m}$ & $\mathrm{O}$ & $\mathrm{u}$ & $\mathrm{t}$ & b & & & $\mathrm{h}$ & $\mathrm{n}$ & $r$ \\
\hline è & e & e & $r$ & 1 & $\mathrm{i}$ & $\mathrm{O}$ & & & $\mathrm{O}$ & $\mathrm{n}$ & $\mathrm{a}$ \\
\hline $\mathrm{S}$ & & & e & $\mathrm{a}$ & $\mathrm{O}$ & 1 & & & $r$ & $\mathrm{i}$ & $\mathrm{i}$ \\
\hline e & & & & $\mathrm{t}$ & $\mathrm{n}$ & e & & & $\mathrm{e}$ & $\mathrm{f}$ & $\mathrm{S}$ \\
\hline & & & & $\mathrm{i}$ & & & & & & $\mathrm{i}$ & $\mathrm{O}$ \\
\hline & & & & $\mathrm{O}$ & & & & & & $\mathrm{c}$ & $\mathrm{n}$ \\
\hline & & & & $\mathrm{n}$ & & & & & & $\mathrm{a}$ & \\
\hline & & & & & & & & & & $\mathrm{t}$ & \\
\hline & & & & & & & & & & 1 & \\
\hline & & & & & & & & & & $\begin{array}{l}\mathrm{O} \\
\mathrm{n}\end{array}$ & \\
\hline
\end{tabular}

De tout ce qui précède, nous déduisons ce que Berranger avait signalé sur l'image poétique chez les surréalistes, et qui diffère entièrement de l'image poétique traditionnelle: "l'image surréaliste naît moins d'une vision préalable retranscrite, ce qui la ramènerait à une mimesis d'hallucination, qu'elle ne donne a voir. [...] cela implique toute une poétique de l'image, qui, au lieu de consigner une vision, ouvrirait sur un nouvel état de la vue, changerait le regard de l'Autre." (BERRANGER 1997, 129). 


\section{Conclusion}

Cette recherche nous semblait autrefois irréalisable à cause de plusieurs points auxquels on s'est heurtée: le sujet, le corpus et les références.

Le sujet, puisqu'il s'agit d'une difficulté redoublée par le choix d'investiguer la poésie engagée mais du point de vue surréaliste. Le corpus nous a représenté un défi, tandis que la production des deux poètes est fertile et dense, donc la difficulté réside dans l'aventure d'opter pour des poèmes intensifiant la vision et l'art des deux poètes d'une manière succincte et précise. Parmi les défis concernant le corpus, on ne trouve aucune traduction française de la poésie de Matar; ce qui augmente la difficulté de la tâche. Les références pour les deux poètes ne sont pas suffisamment disponibles, en particulier en ce qui concerne Mohamed fifi Matar, dont les travaux restaient censurés et interdits en Egypte pour longtemps. Les études sur les deux sont rares, surtout dans les critiques techniques et les explications littéraires.

Malgré cette lacune, le défi devient plaisir, et les clés de les comprendre se révèlent l'une après l'autre. C'est avant tout grâce à la ressemblance idéologique entre les deux, les points communs qui les réunissent, et l'esprit résistant dominant leur poésie engagée. 


\section{Endnotes :}

(1) L'appellation du vers libre commence en 1588 avec Blaise de Vigenère.

(2) La différence, à ce propos, entre le poème en prose et la prose poétique est comme la différence entre la poésie et la prose. Mais les jeux de sonorités dans les deux sont travaillés d'une manière exagérée dans la première, pour compenser l'absence de la rime et du mètre, et dans la deuxième d'une manière la distinguant de la prose ordinaire.

(3) Poète libanais.

(4) Philosophe grec présocratique et connu pour être à l'origine de la théorie cosmogonique des quatre éléments classiques.

(5) C'est à l'opposition du pain noir, fait de farine et de seigle, de sarrasin et de froment, que les Résistants mangent pendant l'occupation. Cela devient un symbole de la pauvre nourriture.

(6) Il s'agit du second accord-cadre rejeté par la quasi-totalité des pays arabes, et qui ne débouchera que sur une paix froide et séparée. L'Égypte est isolée pour ne pas mettre en avant les intérêts arabes et en particulier la reconnaissance des droits des Palestiniens à l'autodétermination.

(7) C'est connu en Egypte comme al-fath al-islaami. Une expression qui montre les expansions de l'empire musulman commençant aux années 630.

(8) Le nom d'un oiseau légendaire très populaire dans la mythologie du Moyen-Orient, en particulier dans les contes de Mille et une nuits. Aussi appelé Rukh. 


\section{Bibliographie}

\section{Corpus}

- ELUARD, Paul. Liberté. Paris: Flammarion, 2012.

- MATAR, Mohamed Afifi. Euvres complètes II. Al-Hay'a AlAmma le-Qossour al-Thaqaafa, 2011.

\section{II.Ouvrages critiques}

- BERRANGER, Marie-Paule. Le surréalisme. Paris: Hachette, 1997.

- COMBE, Dominique. Les genres litteraires. Hachette, 1992.

- GENETTE, Gérard. Figures I. Seuil, 1966. — Figures III. Seuil, 1972.

- REBOUL, Olivier. Introduction à la rhétorique. PUF, 1991.

- TODOROV, Tzvetan. "Synecdoques." In Sémantique de la poésie, by Todorov et autres. Seuil, 1979.

\section{Thèses}

- ABD-ALLAH, Amal ABDEL-SATTAR. "Vers une Démarche Innovatrice du Langage Poétique Français et Égyptien Contemporains." la faculté Al- Alsun, Université de Minia, 2013. 
- LEMJADI, Soureyya. "Signes de métaphore dans la poésie de Muhammad Afifi Matar." Université d'Oran, 2011.

\section{Articles parus dans des périodique}

- ABDEL-MOTTALEB, Mohamed. ""Entrevue avec Afifi Matar dans sa maison poétique"." Al-Kalimah, avril 2009, 28 ed.

- Al-MAGRAHI, Nasser Salem. "'Le poète qui précède le poème d'un pas, Mohamed Afifi Matar"." Almothaqaf, 2021, 5472 ed.

- AZZARRA, Abdou. Al-Ahram, juillet 10, 2020.

- BASTAOUISSI, Ramadan. ""Une expérience philosophique dans la poésie arabe"." Al Kalimah, avril 2009, 28 ed.

- BAZIH, Chaouqi. "Mohamed Afifi Matar." Ach-Charq AlAoussate "Le Moyen Orient", mars 11, 2020, 15079 ed.

- EL-KAFRAOUI, Said. ""Soleil dans le ciel du cœur: Afifi Matar et ouverture aux mondes d'une étrange poésie"." Al-Fayssal, juillet $1,2020$.

- HAMDI, Baligh. ""Matar, le découvreur de la momie brutale"." Acharq Al-Aoussat "Le Moyen Orient", juillet 28, 2018.

- HEROUT, Raphaëlle. "L'imaginaire linguistique du surréalisme." Mélusine, décembre 17, 2016.

- ZAKAREYA, Ahmad. ""Mohamed Afifi Matar, un rapport au Nil"." Jehaat, juin 28, 2014. 


\section{V.Dictionnaires}

- ROBERT, Paul. "Dictionnaire le nouveau petit Robert." Dictionnaire le Robert, 1993.

\section{Sites internet}

- AD-DIKE, Yusuf.

https://montada.aklaam.net/showthread.php?t=5045. 2021. https://montada.aklaam.net/showthread.php?t=5045 (accessed 2021).

- ELUARD, Paul. www.eluardexplique.free.fr. juillet 30, 2021. www.eluardexplique.free.fr.

- MATAR.https://konouz.com/ar/ (accessed 2021).

- VIOUX, Amélie. "Commentaire composé." 2021. https://commentairecompose.fr/liberte-paul-eluard-analyse/. 
الابتداع في الثعر الحديث الملتزم: إيلوار ومطر أنموذجا (دراسة تحليلية) إعداد

د. أمل عبدالستار عبدالله عبدالكريم

مدرس بقسم اللغة الفرنسية وآدابها

كلية الألسن - - جامعة المنيا

الملخص باللغة العربية

كان للأدب- وخاصة الثعر الملتزم- طريقته الخاصة في الدفاع عن القضايا

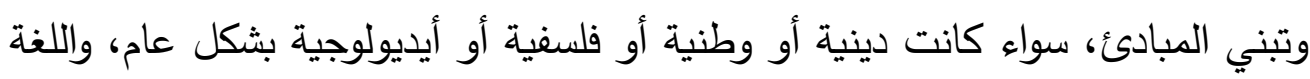
المستخدمة لها قيمة تختلف باختلاف الكلام الموجه، فالسريالية- كاتجاه أدبي وشاعري- لا تتباطأ في وضع روحها وسمتها في الاتجاه الملتزم. لقد استخدمت وسائلها الخاصة، لغتها الخاصة، لإرسال رسالتها السامية إلى

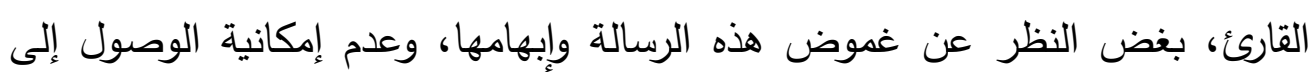

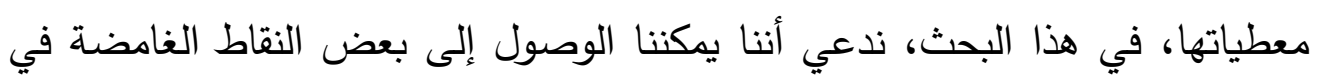
هذا العالم بمساعدة المفاتيح التي استخدمها الثعراء السرياليون؛ وسنكتشف الوسائل

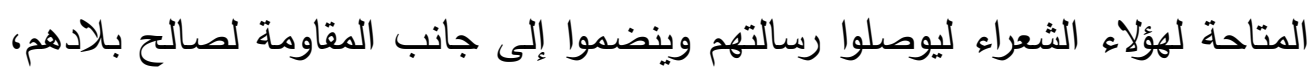
يسهم شكل القصائد ومضمونها بقوة في نقل هذا العالم الغامض والدغلق إلينا، وزن

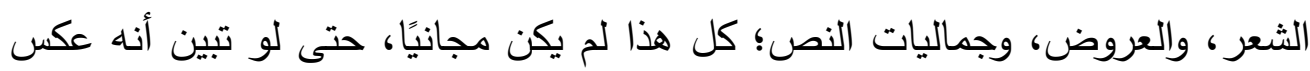

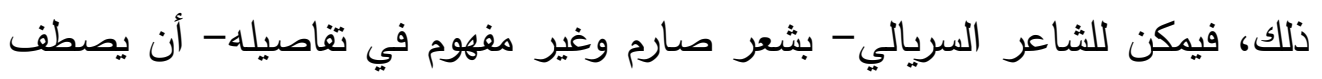
على أنه رجل مبادئ ومقاومة بامتياز . الكلمات المفتاحية: شعر ملتزم، السريالية، إلوار، عفيفي مطر، شعر مبهم، الإغلاق

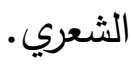

